\title{
Desmontando "mitos" de y desde la patologia bilingüe
}

\author{
FAUSTINo DiÉGuez Vide \\ Universitat de Barcelona \\ Facultat de Filologia i Comunicació \\ Gran Via de les Corts Catalanes, 585 \\ 08007 Barcelona \\ E-mail: fdieguez@ub.edu
}

\section{DESMONTANDO “MITOS" DE Y DESDE LA PATOLOGÍA BILINGÜE}

RESUMEN: En el presente manuscrito se pretende matizar -mediante casos prácticos- tres aseveraciones realizadas en la literatura afasiológica dentro de la patología bilingüe y que se pueden llegar a considerar como "axiomas" (o "mitos"). La primera (qué importancia tiene la distancia estructural en el patrón de recuperación selectivo) se describirá desde la patología agramática en lenguas distantes. La segunda (qué variables se deben considerar más importantes para la patologia bilingüe) se evaluará desde la heterogeneidad observada en algunos pacientes con enfermedad de Parkinson. Por último, la tercera (qué papel juega la exposición al entorno y el hemisferio derecho) se definirá a partir de un caso de recuperación paradójica. Como corolario se intentará ofrecer un panorama que recoja, a partir de los modelos existentes en la actualidad (el modelo declarativo-procedimental y el modelo de red compartida), los elementos fundamentales para realizar un diagnóstico preciso en casos de sujetos bilingües.

PALABRAS ClAVES: bilingüismo; agramatismo; Parkinson; recuperación paradójica; diagnóstico.

SUMARIO: 1. Introducción: acerca de la definición de "mito". 2. Primer "mito": el caso del bilingüe subordinado... o de los agramáticos distantes. 3. Segundo "mito": el caso de las variables variables... y la enfermedad de Parkinson. 4. Tercer "mito": el caso de la recuperación selectiva hacia la L2... o recuperación paradójica. 5. Conclusión: ¿es posible un consenso comunicativo?

\section{DISMANTLING "MYTHS" OF AND FROM BILINGUAL PATHOL- OGY}

ABSTRACT: In the present manuscript, it is intended to clarify -by means of practical cases- three assertions made in the aphasiological literature within the bilingual pathology that can be considered as "axioms" (or "myths"). The first one (how important structural distance is in the pattern of selective recovery) will be described from agrammatic pathology in distant languages. The second one (which variables should be considered more important for bilingual pathology) will be evaluated from the heterogeneity observed in some patients with Parkinson's disease. Finally, the third one (what role plays the exposure to the environment and the right hemisphere will be defined from a case of paradoxical recovery. As a corollary, an attempt will be made to offer a panorama that gathers, from the current models (the declarativeprocedural model and the shared network model), the main elements to make an accurate diagnosis in cases of bilingual subjects.

KEY WORDS: bilingualism; agrammatism; Parkinson; paradoxical recovery; diagnosis.

SUMMARY: 1 . Introduction: About the definition of "myth". 2. First "myth": The case of the subordinate bilingual ... or of distant agrammatics. 3. Second "myth": The case of variable variables ... and Parkinson's disease. 4. Third "myth": The case of selective recovery towards L2 ... or paradoxical recovery. 5. Conclusion: Is a communicative consensus possible?

\section{DEMONTANT DES "MYTHES" DE ET À TRAVERS LA PATHOLOGIE BILINGUE}

RÉSUMÉ: Dans le présent manuscrit, il est prévu de clarifier en utilisant des cas pratiques- trois affirmations faites dans la littérature aphasiologique au sein de la pathologie bilingue pouvant être considérées comme des "axiomes" (ou "mythes"). La première (l'importance de la distance structurelle dans le schéma de récupération sélective) sera décrite à partir de la pathologie agrammatique dans des langues distantes. La deuxième (quelles variables devraient être considérées comme les plus importantes pour la pathologie bilingue) sera évaluée à partir de l'hétérogénéité observée chez certains patients atteints de la maladie de Parkinson. Et pour finir, la troisième (quel rôle joue l'exposition à l'environnement et à l'hémisphère droit) sera définie à partir d'un cas de rétablissement paradoxal. En corollaire, on essaiera d'offrir un panorama rassemblant, à partir des modèles actuels (modèle déclaratif-procédural et modèle de réseau partagé), les éléments fondamentaux pour élaborer un diagnostic précis dans des cas de sujets bilingues

MOTS CLÉS: bilinguisme; agrammatisme; maladie de Parkinson; retablissement paradoxal; diagnostic.

SOMMAIRE: 1. Introduction: à propos de la définition du "mythe". 2. Premier "mythe": le cas du bilingue subordonné... ou des agrammatiques lointains. 3 Deuxième "mythe": le cas des variables variables ... et la maladie de Parkinson. 4. Troisième "mythe": le cas de la récupération sélective vers L2 ... ou de la récupération paradoxale. 5. Conclusion: un consensus communicatif: est-ce posible?
Fecha de Recepción Fecha de Revisión Fecha de Aceptación Fecha de Publicación
$05 / 02 / 2019$

$14 / 03 / 2019$

$17 / 05 / 2019$

$01 / 12 / 2020$ 


\title{
Desmontando "mitos" de y desde la patologia bilingüe ${ }^{1}$
}

\author{
FAUSTINo DiÉGUEZ VIDE
}

\section{INTRODUCCIÓN: ACERCA DE LA DEFINICIÓN DE “MITO”}

Pese a que la etimología asume que un mito se asocia a una fábula o a una leyenda (Corominas, 1961), el DRAE, en su acepción tercera de su primera entrada, sentencia que un mito puede ser una "Persona o cosa rodeada de extraordinaria admiración y estima". Lejos, entonces, de narraciones maravillosas, de historias ficticias, de personajes literarios o... de aves paseriformes, el contexto presente se focaliza en lo admirable y estimable de ciertas personas y de ciertas cosas. Más en concreto, se persigue el objetivo de recoger tres aserciones, casi convertidas en axiomas (y de aquí lo admirable y estimable), y vislumbrarlas a la luz de algunas investigaciones recientes.

Aunque se trate de matices, en ningún caso se trata de criticar posturas de reconocidos neurólogos o psicólogos (o lingüistas), sino más bien de ver con resultados actuales aquellas concepciones que, si bien continuarán siendo correctas, deberian nivelarse levemente. Además, y se reitera el no entrar en anacronismos, esta visión se realizará desde la patología bilingüe -trilingüe o políglota- ${ }^{2}$, pues en estos cuadros patológicos existen ciertas variables, relevantes para el diagnóstico, que nunca se tienen en cuenta en sujetos monolingües. Esto es así porque algunas de estas variables se "presuponen" en un sujeto monolingüe: es obvio que la adquisición de una L1 que diremos lengua materna, al decir de Ribot (1881), y lengua de más uso, al decir de Pitrés (1895)- será temprana y si el sujeto la utiliza a diario también poseerá una alta competencia en su uso. Pero cuando entra en juego una L2 sí es posible encontrar disparidades: adquisición temprana o tardía, competencia alta o baja, método de adquisición formal o informal, etc. Como son muchas las variables que entran en juego y como no existe un consenso sobre cuáles son más significativas y cuáles no -o, lo que es peor, como ninguna justifica todos los casos publicados-, lo que hace que una lengua se recupere y otra no lleva inevitablemente a los mitos-leyendas (GómezRuiz, 2009).

Una ojeada a internet -o a trabajos más científicos, como el de Grosjean (2010)- permite observar que circulan numerosos mitos-leyendas en relación con el bilingüismo, aunque no todos ellos se relacionan con la patología. Estos podrian clasificarse en tres grandes ámbitos. En primer lugar, algunos mitos "generalistas" que van desde los prejuicios lingüísticos ("hay lenguas fáciles y lenguas difíciles") a los politicos ("hay más monolingües en el

\footnotetext{
1 Este trabajo cuenta con la ayuda del Ministerio de Economía y Competitividad [FFI201678034-C2-1-P].

2 Salvo que no se detalle, todo lo que se comente respecto a la patología bilingüe será aplicable, también, a sujetos trilingües o poliglotas. No se hará, entonces, una distinción precisa, salvo en el primer "mito" que se describirá más adelante.
} 
mundo") pasando por los peyorativos ("ser bilingüe no es común o natural: es raro", "el bilingüismo puede provocar trastornos en la socialización y asimilación cultural".

En segundo lugar, hay un conjunto que se relaciona claramente con el mundo infantil. En este caso, la mayoria entra en lo que anteriormente se ha calificado como peyorativo, por no decir ridiculo: "Aprender dos lenguas confunde al niño y reduce su capacidad cognitiva", "La educación bilingüe no es eficaz. Sólo es un obstáculo para el buen aprendizaje de la lengua materna y ralentiza el funcionamiento académico de los alumnos", "Aprender otro idioma puede hacer que el cerebro se sienta sobrecargado, que el aprendizaje sea más lento y que se retrase el desarrollo del lenguaje", "Hablar dos idiomas frente a un niño puede provocar un trastorno del habla o del lenguaje", entre otros.

Por último, hay otro conjunto destinado a los adultos, que se centran en aspectos como la dificultad de un adulto para aprender una lengua (siempre en comparación con un niño), la imposibilidad de hablar una segunda lengua sin acento o la presencia de biculturalidad. No obstante, también hay otros menos válidos: "los bilingües son traductores innatos", "mezclar idiomas es una señal de vaguedad en los bilingües", "los bilingües tienen doble o dividida personalidad", se cuentan entre estos.

Más allá de estos mitos, y retomando la definición de "Persona o cosa rodeada de extraordinaria admiración y estima", el objetivo de este manuscrito se vincula claramente con algunas aseveraciones respecto a ciertas variables que presentan algunos sujetos bilingües en casos patológicos. Si bien se afirma aquí que estas aseveraciones son ciertas o posibles, en algunos casos deberian matizarse con el objeto de englobar a todos los cuadros clínicos. Por supuesto, este matiz se introducirá desde casos prácticos, tanto provenientes de la literatura afasiológica como propios.

\section{PRIMER "MITO": EL CASO DEL BILINGÜE SUBORDINADO... O DE LOS AGRAMÁTI- COS DISTANTES}

El diagnóstico de sujetos bilingües en cualquier clase de patología verbal se vuelve, hoy en día, crucial. Es un hecho demostrado que más de la mitad de la población mundial es bilingüe, trilingüe o poliglota (Weekes, 2010). Además, el número de sujetos bilingües implicados en una patología verbal va en aumento: Paradis consideraba, en el año 2001, que se producen 45.000 nuevos casos cada año en Estados Unidos (número que debe ser similar en Europa). Por supuesto, si este diagnóstico es fundamental, no lo es menos disponer de herramientas terapéuticas conformes a las necesidades de los pacientes, pero, tal y como se ha comentado unos párrafos atrás, es dificil establecer esas herramientas debido a la gran heterogeneidad de variables que entran en juego en una L2.

También es un hecho demostrado que tras una lesión cerebral que afecte a las lenguas de un sujeto bilingüe, se pueden dar numerosos patrones de 
recuperación (cf. Paradis, 1977, para una revisión). Dos de estos patrones son el diferencial y el selectivo, patrones relacionados porque, en ambos casos, una de las lenguas se pierde, parcial (diferencial) o casi totalmente (selectivo). Y no sería temerario aventurar que estos pacientes, en relación con la nueva competencia adquirida tras la lesión (media o baja), pertenecerían a los denominados sujetos bilingües subordinados.

La subordinación, a partir de la competencia en una L2 es un término acuñado por Weinreich, en 1953, que se une a los términos de bilingüismo coordinado y bilingüismo compuesto. Estos dos últimos términos hacen referencia a sujetos bilingües con una alta competencia en la L2: se considera bilingüe coordinado a aquella persona que posee dos sistemas lingüisticos diferentes, cada uno con su propio sistema semántico (en términos saussureanos podríamos decir que son dos significantes y dos significados separados); por su parte, se habla de bilingüe compuesto cuando el sujeto posee dos sistemas lingüísticos, pero solo uno semántico (dos significantes que se corresponden con el mismo significado).

No obstante, el término bilingüe subordinado se reserva a sujetos con una baja competencia en la L2, de manera que se actúa en esta L2 gracias al apoyo en la L1; en otras palabras, el sujeto se apoya en su L1 para poder actuar en su L2. Este apoyo, aplicado a la patología, se vincularía de forma muy clara con los fenómenos paliativos que defendía Nespoulous (1985, 1990). Según este autor, todos los cuadros clínicos incluyen tres fenómenos: negativos (qué ha perdido el paciente: punto de vista clásico en afasiologia), positivos (qué tiene preservado el paciente: perspectiva cognitiva) y paliativos (cómo se puede paliar aquello negativo desde lo positivo). Subordinación y paliación, de esta forma, serian dos caras de la misma moneda en muchas alteraciones verbales. Este uso de estrategias paliativas de copia de la L2 hacia la L1, al menos sintáctica y léxicamente, lo ha puesto de manifiesto Murphy (2003), y se ha observado incluso en lenguas estructuralmente distantes como el chino y el inglés (Luke et al., 2002; Tan et al., 2003).

Este es, precisamente, el objetivo de este apartado: matizar que muchos pacientes con patrones diferenciales o selectivos no pueden considerarse subordinados y que la distancia estructural puede ser la causa de la imposibilidad de "copiar". Este sería el mito: muchos pacientes con patrones diferenciales o selectivos palian su déficit apoyándose en la lengua preservada.

Para esta matización, se describirá brevemente un paciente, WL, un caso que se publicó hace unos años (Diéguez-Vide et al., 2012). En la tabla 1 se muestran las características lingüísticas de WL, un varón nacido en China y hablante nativo de chino mandarín (dialecto You Xian de la provincia de Fujian) ${ }^{3}$ :

\footnotetext{
${ }^{3}$ En la escuela también estudió inglés, pero como no se realizó el estudio de esta lengua se ha eliminado de las características lingüisticas del paciente.
} 


\begin{tabular}{|l|l|l|l|}
\hline & $\begin{array}{l}\text { Chino mandarin } \\
\text { (L1) }\end{array}$ & Castellano (L2) & Catalán (L3) \\
\hline Edad de adquisición & Lengua nativa & $\begin{array}{l}\text { 9 años (inicio en } \\
\text { China) }\end{array}$ & 16 años \\
\hline Competencia & Alta & Media & Baja \\
\hline $\begin{array}{l}\text { Método de adquisición/ } \\
\text { aprendizaje }\end{array}$ & Informal & Formal & Formal/informal \\
\hline $\begin{array}{l}\text { Uso y exposición a la } \\
\text { lengua }\end{array}$ & Familia & $\begin{array}{l}\text { Mass media y algu- } \\
\text { nos amigos }\end{array}$ & $\begin{array}{l}\text { Mass media, es- } \\
\text { cuela y algunos } \\
\text { amigos }\end{array}$ \\
\hline
\end{tabular}

Tabla 1: Características lingüísticas de WL

El día 1 de mayo de 2003, con 20 años, WL presentó de forma brusca un cuadro ictal (pérdida del habla y paresia derecha) con pérdida de consciencia, quedando en coma (Glasgow inicial de 6). Se le realizó un TAC y se observó un hematoma intraparenquimatoso frontoparietal izquierdo con efecto de masa (afectando el centro semioval, la corona radiada, el brazo posterior de la cápsula interna y la cápsula externa).

En el año 2004 se inició el examen neuropsicológico y, lingüísticamente, se administró un examen de chino y catalán con el TAB -Test de Afasias para Bilingües- (Paradis y Libben, 1987), mientras que se utilizó el Test Barcelona (Peña-Casanova, 1991) para el castellano. Los resultados, que aquí resumimos y que pueden ampliarse en Diéguez-Vide et al. (2012), mostraron una clara tendencia en las tres lenguas. En primer lugar, los resultados en la L1 (chino mandarin) fueron totalmente exitosos y WL apenas hizo errores en ninguna de las categorias. Sí es cierto que presentó un problema importante, pues tenía numerosas dificultades para escribir en chino con el alfabeto kanji; sin embargo, en aquel momento no se pudo trabajar este aspecto.

En segundo lugar, en castellano la actuación fue correcta en tareas de lectura y escritura, pero presentaba numerosos déficits en tareas de producción y comprensión orales (una actuación del 50\% en comprensión de órdenes), además de repetición (para pares de palabras, pseudopalabras y oraciones) y denominación (imágenes y partes del cuerpo, pero, sobre todo, en tareas de denominación verbo-verbal y fluencia).

Por último, la actuación en catalán estaba profundamente alterada, salvo algunos items en la lectura y la escritura. El propio paciente demandaba hablar en castellano al presentar dificultades claras en la producción oral.

A pesar de estas actuaciones, donde realmente se observaron aspectos interesantes fue en el ámbito de la fluencia verbal (no se muestra aquí la producción oral en la lengua china porque, tras una revisión por parte de un especialista externo, se aseguró que era completamente normal). Se ofrece a continuación la descripción de la lámina del Test Barcelona en castellano y la denominación de la historieta del TAB en catalán:

1. Lámina de la sala de estar (TB): 
En este cuadrado mujer está leyendo un novela ... y ... parece ... nos parece muy interesante y ... al la esquina hay un. Niño que está buscando ... libro pero está en muy arriba ... está ... un hom ... otro hombre ... no sé qué está haciendo, y el niño está punto de caer ... silla pequeña y los ... jay! ... los padres ... no sé ... senti ... jay! no sé cómo se llama esto, no me sale [ayuda del examinador] ¡Ah sí!, y luego un gato está conseguido un rata negra y el lámpara está ... punto de caer.

\section{Historieta (TAB):}

Un dia ... un matrimonio ... ai!, pist, cómo se llama esto? ai, ai!, sobre l'arbre hi ha un ... [examinador: niu d'ocells] el noi vull ... vol ... gafal els petits, però de repent, l'arbre se lompió i el noi cau ... doncs ... la noia llama a una casa cerca d'allò, llama el teléfono a l'hospital i ambulància, cinc minuts després l'ambulància el porta l'hospital.

Un análisis del texto revela la presencia de muchas palabras castellanas en la historieta en catalán, así como alguna pronunciación anómala. Por supuesto, hay numerosos casos de palabras cognadas que no permiten valorar una posible subordinación del catalán hacia el castellano. Ahora bien, el rasgo más predominante en ambos textos es el agramatismo que se observa tanto en un habla no-fluida (y muy lenta) como en la omisión de diversas palabras gramaticales y en los cambios en la morfología verbal, lo que provoca, en ocasiones, la elisión del verbo.

Retomando ahora la idea principal de este apartado, se aprecia cuál es la dificultad de WL, como bilingüe subordinado en catalán y castellano, para paliar, gracias a la copia de la L1, su problema. Lo apuntaba Paradis:

" [...] we may expect that, given the structural characteristics of specific languages, some of these [agrammatic] manifestations will be more or less salient. At the very least, in a language that does not contain particular items (e.g., definite articles), aphasic patients will not have the opportunity to drop them in obligatory contexts [...] Speakers of languages that are typologically different may be expected to exhibit symptoms that vary in accordance with the parametric differences of their language" (Paradis, 2001: 3).

En otras palabras, WL no puede "copiar" de su L1 para paliar el agramatismo en castellano y/o catalán porque su L1 no posee ni palabras gramaticales ni morfemas, al menos en el sentido morfosintáctico de sus L2 y L3. Se trataria, entonces, de un sujeto bilingüe subordinado en el que los fenómenos positivos de su L1 no permiten paliar los fenómenos negativos de sus L2 y L3. Sí se observa la "copia" de su L2 (castellano) respecto de su L3 (catalán), pues en este caso la distancia estructural entre las lenguas es minima. De hecho, Goral et al. (2006) describieron un caso, EC, trilingüe de 
hebreo (L1), inglés (L2) y francés (L3) en el que se produjeron resultados similares a los aquí descritos, causados igualmente por la distancia estructural entre lenguas.

Esta distancia psicotipológica (Murphy, 2003), junto con alguna de las variables comentadas, podría llevar a la conclusión de que existen diferentes sustratos neuronales para las lenguas. En este sentido, Ansaldo et al. (2008) han argumentado que una adquisición temprana e informal imbricaría la L1 a la memoria implicita, mientras que una adquisición tardía y formal lo haría respecto a la memoria explícita. Pero se volverá sobre este tema en los otros casos descritos al hilo de algunos modelos actuales.

\section{SEGUNDO "MITO": EL CASO DE LAS VARIABLES VARIABLES... Y LA ENFERME- DAD DE PARKINSON}

Es necesario, antes de afrontar este segundo matiz, ofrecer una breve justificación de este título doble, pues tiene que ver tanto con la reduplicación como fenómeno enfático como con la presencia de un nombre seguido de un adjetivo. Esto significa que muchas de las variables que la literatura ha descrito para la recuperación de una L2 serian obligatorias mientras que otras se podrian considerar como optativas.

Y este es, claro está, el mito: hay variables significativas en el diagnóstico de sujetos con patología bilingüe y variables poco relevantes.

Es posible ilustrar, con tres ejemplos, el tipo de variables que se postulan cuando se recupera una lengua.

1) Fabbro (1999): grado de familiaridad de una lengua, factores visuales (métodos formales), factores psicológicos y emocionales, lengua hablada por el personal del hospital, lengua que se usa en circunstancias especiales, lengua aprendida en la universidad, factores lingüísticos (distancia estructural) y factores orgánicos (edad del paciente, tipo de lesión, etc.).

2) Piske et al. (2003): edad de adquisición, lugar de residencia (tipo de exposición), género, métodos formales, motivación, aptitud para aprender una lengua, uso de la lengua.

3) Murphy (2003): competencia, uso y exposición, modo lingüístico (monolingüe vs. bilingüe), conciencia lingüística, edad, conocimientos académicos, contexto, tipología (distancia estructural), frecuencia, categoria gramatical, morfología.

Es cierto que muchas de las diferencias en las listas anteriores se deben al tipo de investigación que es, por orden, el siguiente: libro de divulgación, investigación sobre el acento, investigación sobre la transferencia de una L2 mientras se adquiere una L3. Pero más allá de esto, lo que es realmente interesante es que todas estas variables se han propuesto desde investigaciones o estudios, es decir, que ninguna de ellas es puramente teórica. 
¿Qué variables se deben postular, entonces, como prioritarias? Durante algún tiempo, se propusieron esencialmente tres variables como las más importantes o significativas respecto al resto. Una de estas tres, la edad de adquisición, que divide a los sujetos en tempranos o tardíos -si la adquisición no supera o supera, respectivamente, el período crítico-, se reafirmó como prioritaria. Existen diversas investigaciones en las que se afirma que una producción oral nativa (acento) en L2 sería (casi) imposible, incluso en sujetos que hubieran tenido contacto con dos lenguas desde la infancia (Scovel, 1988; Sebastian-Gallés et al., 2005), mientras que sería imposible en sujetos con adquisición tardia (Sebastian-Gallés et al., 2006; Simmonds et al., 2011).

Otra de estas variables es la competencia que se vincularía especialmente con el uso premórbido de las lenguas. Es evidente que cuanto más uso, más competencia. Una alta o baja competencia podria ser también un factor clave a la hora de recuperar una lengua tras una lesión.

Por último, la tercera variable se relacionaría con el método de adquisición de la L2, pues este puede ser informal (especialmente mediante inmersión lingüística) o formal (esencialmente, en la escuela)

Para valorar la importancia de estas variables, vale la pena observar los primeros trabajos realizados con pacientes de Parkinson bilingües. El grupo de Zanini y Fabbro (Zanini et al., 2004, 2010; Johari et al., 2013), controlaron, precisamente, estas tres variables, como se puede apreciar en la tabla 2:

\begin{tabular}{|l|l|l|l|}
\hline Estudio & $n$ & L1 & L2 \\
\hline $\begin{array}{l}\text { Zanini et } \\
\text { al. (2004, } \\
2010)\end{array}$ & 9 & $\begin{array}{l}\text { - Friuliano. } \\
\text { U Uso en el entorno fami- } \\
\text { liar }\end{array}$ & $\begin{array}{l}\text { - Italiano. } \\
\text { Adquisición formal (escuela): adquisición } \\
\text { tardía. } \\
\text { - Uso en el trabajo: alta competencia. }\end{array}$ \\
\hline $\begin{array}{l}\text { Johari et } \\
\text { al. (2013) }\end{array}$ & 13 & $\begin{array}{l}\text { - Azari (azerbaiyano). } \\
\text { - Uso habitual. }\end{array}$ & $\begin{array}{l}\text { - Farsi. } \\
\text { - Adquisición formal (escuela): adquisición } \\
\text { tardía. } \\
\text { - Uso habitual: alta competencia. }\end{array}$ \\
\hline
\end{tabular}

Tabla 2: Características lingüísticas de las primeras investigaciones con pacientes de Parkinson bilingües

Como muestra la tabla, las características en relación con el bilingüismo son muy similares (casi simétricas, exceptuando, claro está, las lenguas) y, lo que es más interesante, es que solo se controlaron, en un inicio, estas variables. Se comenta también en un pequeño párrafo que las dos lenguas, en ambos casos, son distantes estructuralmente, pero es un dato que no se tuvo en consideración a la hora de discutir los resultados (es quizás una variable que se vinculaba más a la terapia que al diagnóstico, como se apuntó en el apartado precedente).

Los resultados obtenidos mostraron una peor actuación tanto en la L1 como en la L2 respecto a los sujetos control, pero, y esto es lo importante, la actuación en L1 y L2 fue también diferencial. En general, en las tareas 
que requerian del uso de "habilidades" (fonologia, morfologia, sintaxis) la actuación fue peor en la L1 por lo que llegaron a la conclusión de que los pacientes de Parkinson muestran una alteración gramatical general en su lengua nativa.

Estos datos permitieron a los autores ir en defensa del denominado modelo declarativo-procedimental, que se comentará al final del manuscrito (ver apartado 5). Pero sí vale la pena describir ahora, con mucha brevedad, que los ganglios basales, lugar de la lesión en los pacientes de Parkinson, jugarían en este modelo un papel clave en la selección de la L2 dependiendo del momento de adquisición.

Pero, ¿cómo se relacionan estos resultados con la relevancia de las variables? Aun en el ámbito de sujetos bilingües con la enfermedad de Parkinson, es necesario también citar otro trabajo, cuyas características se reflejan en la tabla 3:

\begin{tabular}{|l|l|l|l|}
\hline Estudio & $n$ & $L 1$ & $L 2$ \\
\hline $\begin{array}{l}\text { Cattaneo et al. } \\
\text { (2015) }\end{array}$ & 28 & $\begin{array}{l}\bullet \text { Catalán. } \\
\text { - Uso habitual. }\end{array}$ & $\begin{array}{l}\text { - Castellano. } \\
\text { - Adquisición temprana. } \\
\text { - Uso habitual: alta competencia. }\end{array}$ \\
\hline
\end{tabular}

Tabla 3: Características lingüísticas de los pacientes bilingües de Parkinson en la investigación de Cattaneo et al. (2015)

Como se observa en este nuevo estudio, se utilizan las mismas variables que en los estudios anteriores, con la salvedad de que ahora la adquisición de la L2 es temprana. No obstante, en este caso, los resultados fueron diferentes: se observó una actuación con prácticamente la misma tasa de éxito para la L1 y la L2. Sí que se apreció también una tendencia hacia una peor actuación en la L1, pero solo en aquellos contextos en los que el sujeto debía trabajar con tareas específicas (como las de interferencia entre ambas lenguas o las pruebas de fluencia fonémica).

Como en el caso anterior, estos resultados invalidan, según los autores, el modelo declarativo-procedimental y son un apoyo al denominado modelo de red compartida. No obstante, como ya se ha comentado, se pospone la justificación de estos modelos para más adelante (ver apartado 5), aunque se puede comentar ahora que los ganglios basales -y, en particular, el caudado- actúan como una estructura de control para la selección de una lengua, control que será diferencial según la edad de adquisición y el grado de competencia.

A partir de las dos tablas anteriores, alguien podría estar tentado a defender que solo la adquisición de la lengua es la variable importante, pues es la que diferencia unos resultados de otros (más allá de la distancia estructural). No obstante, es necesario también anotar ahora un par de cuestiones: primera, que el periodo critico en los estudios de Zanini, Fabbro y colaboradores era de 6 años (edad escolar); y, segunda, que Cattaneo et al. (2015) apuntan también que la exposición a la lengua sería una variable significativa, ofreciendo algo más de dinamismo a las variables. 
Como es necesario, entonces, valorar estas variables, junto a la exposición, se entrará ahora en el tercer "mito", un mito que imbrica todas las variables comentadas hasta ahora.

\section{TERCER "MITO": EL CASO DE LA RECUPERACIÓN SELECTIVA HACIA LA L2... O RECUPERACIÓN PARADÓJICA}

En 1999, Fabbro definió una recuperación paradójica como el patrón de recuperación en el que se recupera una L2 a expensas de una L1 que es la lengua materna o de más uso. Este patrón se propuso para dos casos: primero, para la recuperación de una lengua muerta (de la liturgia o clásica); y, segundo, para la recuperación de una L2 hablada en la actualidad. Es bastante evidente que una recuperación paradójica sería un subtipo de patrón de recuperación diferencial o, sobre todo, selectivo, pero no entrarian en esta etiqueta los sujetos con el denominado patrón de recuperación antagonista.

Desde el trabajo inicial de Gelb en 1937, han aparecido diversos casos de recuperaciones paradójicas "puras", es decir, con adquisición tardía y baja competencia (Aglioti y Fabbro, 1993; Aglioti et al., 1996; Garcia-Caballero et al., 2007; Leeman et al., 2007). No obstante, se suelen incluir también, al menos con efectos de localización anatómica y modelización, los casos de recuperación selectiva hacia la L2, ya sea con adquisición tardía pero alta competencia, ya sea con adquisición temprana y alta competencia (ver Diéguez-Vide et al., sometido, para una descripción de todos estos casos). Por supuesto, en sujetos trilingües se pueden dar un par de combinaciones de las variables mencionadas. Por su interés respecto a los modelos, se describirán algunos de estos casos en el siguiente apartado.

El objetivo de este apartado es, por supuesto, describir un caso de recuperación paradójica (Diéguez-Vide et al., 2019), con alguna característica especial. El caso concreto es el de una mujer, MASS, de 64 años, nacida en Andalucía y que se trasladó a vivir en Girona a los 20 años. La tabla 4 muestra sus características lingüísticas.

\begin{tabular}{|l|l|l|}
\hline & Castellano (L1) & Catalán (L2) \\
\hline Edad de adquisición & Infancia & 20 años \\
\hline Competencia & Alta & Baja \\
\hline $\begin{array}{l}\text { Método de adquisi- } \\
\text { ción/aprendizaje }\end{array}$ & Informal & Informal \\
\hline Uso y exposición a la lengua & $\begin{array}{l}\text { Mass media, familia, amigos y en- } \\
\text { torno }\end{array}$ & Entorno \\
\hline Tipo de bilingüe & Pasivo (no-equilibrado o no-balanceado) \\
\hline Lengua de instrucción & Iletrada \\
\hline
\end{tabular}

Tabla 4: Características lingüísticas de MASS

Como se puede observar en la tabla 4, MASS encaja en las variables de adquisición tardía y baja competencia (recuperación paradójica), pero se añaden un par de datos interesantes: el método de adquisición para L2 -y, 
por supuesto, L1- es informal (pues es iletrada) y solo se relacionaba en catalán con el entorno, de forma pasiva (es decir, participaba en eventos sociales y realizaba sus actividades diarias oyendo a las personas hablar en catalán, aunque siempre respondía en castellano). De acuerdo con la información que aportaron los familiares, siempre se expresaba en castellano y seguía en esta lengua a los medios de comunicación (especialmente, televisión y radio).

A finales del año 2006 sufre un accidente de tráfico (Escala de Coma de Glasgow: 8) y en un TAC se observó fractura craneal con hematoma subdural temporoparietal izquierdo. Un nuevo TAC dos dias después reveló dos contusiones hemorrágicas agudas en las cortezas temporal y occipital izquierdas y una contusión no-hemorrágica en la circunvolución frontal inferior izquierda. Tras despertar del coma unos dias después, mostró una afasia y una paresia del brazo izquierdo. Pero lo que alarmó a la familia es que solo hablaba en catalán. Incluso, cuando se dirigian a ella en castellano, invariablemente respondía en catalán. ${ }^{4}$

Un análisis neurolingüístico mostró una actuación claramente diferenciada entre la L1 y la L2. En la L1 MASS mostraba una producción oral con una no-fluencia severa (prácticamente mutismo o lenguaje reducido a unas pocas palabras), aunque sí era capaz de repetir y de comprender oralmente. Si bien no se realizó un análisis exhaustivo, podría diagnosticarse como afasia motora transcortical de variante clásica (Diéguez-Vide y Peña-Casanova, 2012). Por su parte, la L2 era fluida y gramaticalmente correcta, con repetición y comprensión correctas, y solo destacaba una ligera anomia, lo que encajaría en el cuadro clínico de afasia anómica.

Para mostrar claramente esta fluencia, se muestra a continuación una transcripción de la lámina "La sala de estar" -adaptada- del Test Barcelona (Peña-Casanova, 1991) (los números entre paréntesis marcan pausas superiores a 3 segundos; menos de 3 segundos se marcan con puntos suspensivos):

\section{Catalán (230 segundos)}

bueno això una pilota, no? [4s] això és lo que es posa... la llum ... però no sé com se diu [8s] no me surt ... això és una dona... un llibre... això és unaaa, com se diu això [6s] una barqueta [9s] una taula... això és un [7s] se posa al terra però no me'n recordo ara... jasana [neologismo]... bueno, no sé... això és unaaa planteta... això és un... lo que se posa al redor per ficar les cosetes que, que treu ay... però no sé com se diu... això és un... mare meva [7s] no sé... una altra dona, o noia, no sé... està agafant els llibres [5s] ay com se diu [8s] ay, jo això ho sé [6s] jo he tingut moltes en casa... me'n sembla que són aquestes... ay [4s] tenia moltes [9s] palomas... això se diu...

\footnotetext{
${ }^{4}$ En un test de denominación, llegó incluso a modificar el género para producir la emisión en catalán. En el test de completamiento denominando, se le ofreció el contexto "Por la noche dormimos en la..." y la respuesta fue "el llit".
} 
i bueno... i això sí que ja no me’n recordo... no me surten... i tinc posades jo també en casa però no, no me surten [4s] bueno [6s] ja està.

\section{Castellano (258 segundos)}

54s] dona, pero no eso no es [risa] ... eso està malament... en castellà [10s] unaaa [22s] señ, señora... ay mare meua... una señora [5s] això que té és un llibre, però en castellà [4s] pues igual... llibre [risa] [interrupción del examinador: intente hablar en castellano] bueno... están las palomas [10s] el pare... par [14s] padre [15s], la filla [interrupción del examinador: en castellano] [19s] en castellano [7s] hija, hija... això és una planteta, és lo mateix? [6s] planta, una planta

Como se observa, el número de palabras es completamente divergente: 56 en la L1 y 182 en la L2. Además, medido en unidades de información (Nicholas, y Brookshire, 1993; Marchina et al., 2011), la emisión en catalán era mucho más informativa (20 unidades) que en castellano ( 7 unidades).

Está claro que en este caso ni la edad de adquisición, ni la competencia, ni el uso justifican estos resultados. De aquí se deduce que solo existe una explicación, al menos para que MASS pueda producir oralmente como lo hacía: la exposición al entorno. Pero esta exposición, que convierte a la paciente en bilingüe pasivo, no es suficiente para explicar cómo es capaz de hablar una lengua que, hasta ese momento, nunca habia utilizado. Se podría dar una respuesta matizando el tercer "mito": Todo el procesamiento gramatical de una lengua está lateralizado al hemisferio izquierdo.

Es bastante poco probable que todo el procesamiento sea izquierdo, al menos desde la perspectiva de dos o más lenguas, pues, como se ha comentado respecto a las lesiones, MASS presentaba contusiones hemorrágicas en las cortezas temporal y occipital, y contusiones no-hemorrágicas en la corteza frontal. La única respuesta plausible a partir de aquí es que gran parte de su procesamiento, al menos para la L2, se localice en el hemisferio derecho. Como prueba de este procesamiento -que va más allá del procesamiento prosódico, léxico-semántico y discursivo en sujetos monolingües (véase Joanette et al., 2008, para una revisión)- existen, al menos, dos estudios (Castro-Caldas y Reis, 2000; Carreiras et al., 2009) en los que se ha mostrado que las personas analfabetas no poseen una lateralización izquierda en todas las tareas lingüísticas. Igualmente, existe evidencia de que sujetos con una adquisición tardía (Wartenburger et al., 2003) o con un nivel muy bajo de competencia (Perani et al., 1996; Dehaene et al., 1997; Ansaldo et al., 2008) muestran activación en la L2 de ciertas regiones del hemisferio derecho, lo que les permite paliar las alteraciones que muestran (quizás gracias a mecanismos pragmáticos). De hecho, Wartenburger et al. (2003) han llegado a afirmar que se produciría un vínculo entre la circunvolución frontal inferior izquierda -incluyendo el área de Broca- y las regiones análogas en el hemisferio derecho cuando se deben procesar estimulos morfosintácticos. 


\section{ConClusión: ¿ES POSIBLE Un CONSENSO COMUNICATIVO?}

Páginas atrás se han descrito, al menos, dos aspectos alrededor de la patología bilingüe: primero, que existe una heterogeneidad en la descripción de la localización de una L1 y una L2; y, segundo, que no existe un acuerdo sobre qué variable o variables son las más significativas. Brevemente, en este apartado, se intentarán matizar los matices... a modo de consenso.

Como se ha comentado anteriormente, existen dos modelos que "pugnan" por la localización de dos o más lenguas. Uno de ellos, el modelo declarativoprocedimental $^{5}$, se basa en la existencia de dos sistemas mnésicos: uno léxico y otro gramatical. El sistema declarativo, vinculado con la memoria semántica y episódica, se relaciona con un aprendizaje consciente y se localizaría en estructuras mediales del lóbulo temporal (hipocampo) conectadas con áreas corticales temporales y parietales en ambos hemisferios. Por su parte, la memoria procedimental o implícita se vincularia con "habilidades" o "hábitos" y, almacenada inconscientemente -automáticamente-, estaría mediada por circuitos fronto-estriados. Esta separación anatómica implicaría, en hablantes monolingües, una separación entre el conocimiento gramatical y el léxico-semántico, estando el primero localizado en la memoria implícita, mientras que el segundo lo hace en la explícita. Esta separación también se produce en sujetos bilingües tempranos (edad de adquisición) y con una alta competencia. No obstante, los casos en los que se trata de sujetos bilingües tardíos y/o con baja competencia, no presentarian el mismo patrón: todo el procesamiento de la L2 estaría localizado en las estructuras temporales y parietales comentadas, pero no en zonas frontales o basales.

La validación de esta propuesta, que favorece a las variables edad de adquisición y competencia, proviene de dos fuentes. En primer lugar, casos de recuperación paradójica con pacientes que presentan lesiones circunscritas a los ganglios basales y que muestran una alteración gramatical en la L1 (Aglioti y Fabbro, 1993; Fabbro y Paradis, 1995; Aglioti et al., 1996; Moretti et al., 2001; García-Caballero et al., 2007; Ansaldo et al., 2008; Adrover-Roig et al., 2011); es necesario comentar que los casos de Fabro y Paradis, y de Adrover-Roig et al., presentaban una adquisición temprana y una alta competencia (patrón de recuperación selectivo hacia la L2), mientras que el resto sí encajan en un patrón paradójico (adquisición tardía y baja/media competencia). En segundo lugar, y como ya se ha comentado, la evidencia se imbrica también con pacientes afectados de Parkinson, pues se trata de sujetos con alteración en los ganglios basales (Zanini et al., 2004, 2010; Johary et al., 2013).

\footnotetext{
${ }^{5}$ Véanse Paradis $(1994,2004)$ y Ullman $(2001 a, 2001 b)$ para una revisión exhaustiva de este modelo.
} 
Por su parte, el denominado modelo de red compartida ${ }^{6}$ sostiene que las mismas estructuras que subyacen a la $L 1$ también lo hacen respecto a la L2. A pesar de que se superpone con el anterior modelo en algunos aspectos (pues participan, entre muchas otras, las mismas estructuras), presenta algunos rasgos fundamentales que lo hacen muy diferente: la noción de control y la participación de procesos de inhibición. Las estructuras de control, que se detallan en Calabria et al. (2018) se describen brevemente a continuación. La corteza prefrontal (y, en especial, la parte dorsolateral) participaría en la resolución de conflictos inhibiendo las respuestas de la lengua no en uso y seleccionando las respuestas de la lengua que se quiere utilizar: la corteza prefrontal izquierda seleccionaría una respuesta, mientras que la derecha inhibiria una respuesta. En este mismo procesamiento, la parte dorsal anterior de la corteza cingular participaria en el control y detección de conflictos.

El lóbulo parietal inferior también participaría en esta red, controlando la selección de la lengua, tanto fonológicamente (circunvolución supramarginal) como semánticamente (circunvolución angular): en el hemisferio izquierdo sesgaría la selección de la lengua que no se usa, mientras que en el derecho haría lo propio con la lengua que se usa.

El núcleo caudado izquierdo, dentro de los ganglios basales, controlaría las dos lenguas evitando interferencias de una hacia la otra, planificando la emisión y seleccionando el candidato léxico más apropiado. Por su parte, el putamen se encargaria de controlar los procesos articulatorios.

Por último, el cerebelo, especialmente el hemisferio cerebeloso derecho, también participaría en esta red manteniendo una representación mientras se realiza la comprensión y ayudando a resolver conflictos en la producción oral.

Todas estas estructuras serian sensitivas, en población bilingüe, a la competencia y a la exposición a la lengua, de manera que la edad de adquisición no sería una variable tan significativa. De esta forma, sujetos con una baja competencia o con una baja exposición a la lengua necesitarán un mayor control para poder inhibir la L2, control que será automático en sujetos con alta competencia o alta exposición. En el caso de los patrones de recuperación selectiva hacia la L2 o paradójica, Abutalebi et al. (2009) proponen que la pérdida de una lengua se vincularía a un umbral de activación elevado para la $\mathrm{L} 1$.

Como en el modelo anterior, la validación ahora procede de pacientes con recuperaciones selectivas hacia la L2 o recuperaciones paradójicas en las que no se aprecian lesiones específicas en los ganglios basales (Ibrahim, 2008; Goral et al., 2013; Lee et al., 2016; Radman et al., 2016), así como del estudio ya comentado con pacientes de Parkinson (Cattaneo et al., 2015).

\footnotetext{
${ }^{6}$ Véase, por ejemplo, Wartenburger et al. (2003); Perani y Abutalebi (2005); Abutalebi y Green (2007); Abutalebi et al. (2009), para una revisión.
} 
Es también necesario ahora comentar que los resultados de Calabria y colaboradores se obtuvieron con sujetos que poseían una adquisición temprana y una alta competencia; por su parte, el estudio de Goral et al. incluía sujetos con adquisición temprana y alta competencia (L2), y con adquisición tardia y baja competencia (L3).

Por supuesto, ambos modelos han intentado rebatir los "puntos débiles" del modelo contrario. Ya se acaban de comentar los casos en los que existe una pérdida de la L1 y preservación de la L2, en sujetos con baja o media competencia, sin lesión en los ganglios basales, unos resultados que no encajarian en las propuestas del modelo declarativo-procedimental. Por su parte, este modelo critica, del modelo de red compartida, la dificultad para explicar ciertos patrones de conducta, aunque, al no ser significativa la variable edad de adquisición, sería muy dificil encontrar sujetos con alta competencia y bajo nivel de exposición. Pero la recuperación paradójica -sujetos con baja competencia y alto nivel de exposición- sigue siendo un aspecto con un encaje complejo en estos modelos, sobre todo si existe la posibilidad de que participe el hemisferio derecho.

¿Es posible, entonces, ofrecer una única respuesta? ¿Cómo encajar todos estos matices? Quizás la respuesta a estos interrogantes podría proceder de la denominada hipótesis del control adaptativo formulada en el año 2013 por Green y Abutalebi. De acuerdo con esta propuesta, existirian tres contextos de interacción bilingües definidos como patrones recurrentes de intercambio comunicativo que reflejan el día-a-día de cada persona:

1) Contexto de lengua única: una lengua se usa en un entorno y la otra lengua se usa en un segundo entorno diferente. Se trata de un contexto en el que no se producen interferencias entre lenguas.

2) Contexto de lengua dual: se utilizan conjuntamente ambas lenguas, pero con hablantes diferentes. Se pueden producir interferencias entre lenguas dentro de la conversación, pero no dentro de las emisiones.

3) Contexto denso de cambio de código: se trata de situaciones comunicativas en las que los hablantes intercambian de código en el transcurso de las diferentes emisiones. En esta situación se producen numerosas interferencias y adaptaciones de palabras de una lengua a otra.

Estos tres contextos, que requieren el uso de mecanismos de control e inhibición específicos como los descritos anteriormente, cubririan los intercambios comunicativos del día-a-día en sujetos bilingües. Dependiendo de la situación, se debe seleccionar una lengua e inhibir una segunda lengua. No obstante, estos tres contextos, tal y como están planteados, poseen una clara limitación: solo cubren la producción oral, pues es el ámbito donde pueden existir interferencias. Imbricándolo con la competencia, una de las variables principales del modelo, cuanta más competencia en la L2 menos interferencias. 
Esta limitación implica que no entren en consideración, sobre todo en la patología bilingüe, los denominados bilingües pasivos, es decir, aquellos sujetos que solo comprenden una lengua que no hablan. Esta diferenciación ya la puso de manifiesto Green (1998) cuando asumía que las interferencias en la producción se podrian deber a un error en los procesos de control de dominio-general, mientras que las observables en la comprensión se vincularían a procesos de control de dominio-específico. Desde esta perspectiva, es natural considerar que, según el grado de exposición -combinado con otros factores- una persona puede permanecer siempre como bilingüe pasivo, como se ha visto en el caso de MASS. Los mecanismos de control propuestos para los tres contextos anteriores, en este caso, funcionarian de forma diferente para un sujeto bilingüe pasivo, pues ese control no podrá nunca ser automático.

En resumen, sin menospreciar -antes, al contrario- los tres contextos de intercambio comunicativo, y manteniendo que podrian ser una herramienta imprescindible en la patología bilingüe, sí que es necesario comentar que se deberian introducir mecanismos para vincular, al menos, tres aspectos:

1) La posibilidad de que el sujeto bilingüe pasivo subordine su lengua no dominante a su lengua dominante (el caso del bilingüe subordinado).

2) La posibilidad de que los contextos comunicativos -y la situación de cada persona- enfatice unas variables por encima de otras, y no siempre las mismas (el caso de las variables variables).

3) La posibilidad de que participen otras estructuras anatómicas en el control comentado y, en casos de sujetos con escasos recursos en la L2, sea el hemisferio derecho el que se encargue de realizar el control sobre los contextos comunicativos (el caso de la recuperación selectiva hacia la L2).

\section{REFERENCIAS}

ABUTALEBI, J. et al. (2009): "Bilingual aphasia and language control: A follow-up fMRI and intrinsic connectivity study", Brain and Language, 109, pp. 141-156.

ABUTALEBI, J. \& GREEN, D. W. (2007): "Bilingual speech production: The neurocognition of language representation and control", Journal of Neurolinguistics, 20, pp. 242-275.

ADROVER-ROIG, D. et al. (2011): "Impaired L1 and executive control after left basal ganglia damage in a bilingual BasqueSpanish person with aphasia", Clinical Linguistics and Phonetics, 25, pp. 480-498.

AGLIOTI, S. \& FABBRO, F. (1993): "Paradoxical selective recovery in a bilingual aphasic following subcortical lesions", Neuroreport, 4, pp. 1359-1362.

AGLIOTI, S. et al. (1996): "Neurolinguistic and follow-up study of an unusual pattern of recovery from bilingual subcortical aphasia”, Brain, 119, pp. 15511564. 
ANSALDO, A-I. et al. (2008): "Language therapy and bilingual aphasia: Clinical implications of psycholinguist-tics and neuroimaging research", Journal of Neurolinguistics, 21, pp. 539557.

CALABRIA, M. et al. (2018): "Neural basis of bilingual language control", Annals of the New York Academy of Sciences, 19, pp. 115.

CARREIRAS, M. et al. (2009): "An anatomical signature for literacy", Nature, 461, pp. 983-986.

CASTRO-CALDAS, A. \& REIS, A. (2000): "Neurobiological substra-tes of illiteracy", The Neuroscientist, 6 , pp. 475-482.

CATTANEO, G. et al. (2015): "The role of executive control in bilingual language production: A study with Parkinson's disease individuals", Neuropsychologia, 66, pp. 99-110.

COROMINAS, J. (1961): Breve diccionario etimológico de la lengua castellana, Madrid: Gredos.

DEHAENE, S. et al. (1997): "Anatomical variability in the cortical representation of first and second language", Neuroreport, 8, pp. 3809-3815.

DIÉGUEZ-VIDE， F. y PEÑA-CASANOVA, J. (2012): Cerebro y lenguaje: sintomatología neurolingüistica, Madrid: Panamericana.

DIÉGUEZ-VIDE, F. et al. (2012): "Chinese-Spanish-Catalan trilingual aphasia: A case study", Journal of Neurolinguistics, 25, pp. 630-641.

DIÉGUEZ-VIDE， F.; FERNÁNDEZPLANAS, A. M.; GICH-FULLÀ,
J.; PUIG, J.; BERTHIER, M. L. \& ELVIRA-GARCÍA, W. (2019): "Paradoxical recovery of L2 in a bilingual aphasic person: adressing issues on causative factors and accent", Aphasiology, https://doi.org/10.1080/0268 7038.2019 .1661956$.

FABBRO, F. (1999): The neurolinguistics of bilingualism. An introduction, Hove: Psychology Press.

FABBRO, F. \& PARADIS, M. (1995): "Differential impairments in four multilingual patients with subcortical lesions", Paradis, M. (ed.): Aspects of Bilingual Aphasia, vol. III, Oxford: Pergamon, pp. 139-176.

GARCÍA-CABALLERO, A. et al. (2007): "Paradoxical recovery in a bilingual patient with aphasia after right capsuloputaminal infarction", Journal of Neurology, Neurosurgery and Psychiatry, 78, pp. 89-91.

GELB, A. (1937): "Zur medizinischen Psychologie und philosophischen Anthropologie", Acta Psychologica, 3, pp. 193-27. [Traducido al inglés en $M$. Paradis (ed.) (1983): Readings on aphasia in bilingual and polyglots, Montreal: Marcel Didier, pp. 383-384].

GÓMEZ-RUIZ, M. I. (2010): "Bilingüismo y cerebro: mito y realidad", Neurología, 25, pp. 443-452.

GORAL, M. et al. (2006): "Cross-language lexical connections in the mental lexicon: Evidence from a case of trilingual aphasia", Brain and Language, 98, pp. 235-247.

GORAL, M. et al. (2013): “Asymmetric inhibitory treatment effects in 
multilingual aphasia”, Cognitive Neuropsychology, 30, pp. 564-577.

GREEN, D. W. (1998): "Mental control of the bilingual lexico-semantic system", Bilingualism: Language and Cognition, 1, pp. 67-87.

GREEN, D. W. \& ABUTALEBI, J. (2013): "Language control in bilinguals: The adaptive control hypothesis", Journal of Cognitive Psychology, 25, pp. 515-530.

GROSJEAN, F. (2010): Bilingual life and reality, Cambridge (Mass.): Harvard University Press.

IBRAHIM, R. (2008): "Performance in L1 and L2 observed in ArabicHebrew bilingual aphasic following brain tumor: A case constitutes double dissocia-tion", Psychology Research and Behavior Management, 1, pp. 1119.

JOANETTE, Y. et al. (2008): "Impacto de las lesiones del hemisferio derecho sobre las habilidades lingüisticas: perspectivas teórica y clínica", Revista de Neurología, 46, pp. 481-488.

JOHARI, K. et al. (2013): "Grammatical deficit in bilingual AzariFarsi patients with Parkinsons disease", Journal of Neurolinguis-tics, 26, pp. 22-30.

LEE, B. et al. (2016): "Recovery of language function in Korean-Japanese crossed bilingual aphasia following right basal ganglia hemorrhage", Neuroca-se, 7, pp. 1-6.

LEEMANN, B. et al. (2007): "Paradoxical switching to a barely-mastered second language by an aphasic patient", Neurocase, 13, pp. 209-213.
LUKE, K. K. et al. (2002): "Functional anatomy of syntactic and semantic processing in langua-ge comprehension", Human Brain Mapping, 16, pp. 133-145.

MARCHINA, S. et al. (2011): "Impairment of speech production predicted by lesion load of the left arcuate fasciculus", Stroke, 42, pp. 2251-2256.

MORETTI, R. et al. (2001): "Bilingual aphasia and subcortical-cortical lesions", Perceptual and Motor Skill, 92, pp. 803-814.

MURPHY, S. (2003): "Second language transfer during third language acquisition", Teachers College, Columbia University Working Papers in TESOL \& Applied Linguistics, 3 (1), pp. 1-21.

NESPOULOUS, J-L. (1985): "Neurosemiotics: structures, processes and strategies", Recherches Semiotiques/SI, 5, pp. 383-392.

NESPOULOUS, J-L. (1990): "Linguistique, neurolinguistique et psycholinguistique: un parcours en quatre étapes", Nespoulous, J-L. \& Leclerq, M. (eds.): Linguistique et neuropsycholinguistique: tendances actuelles, Paris: Société de Neuropsychologie de Langue française, pp. 1-4.

NICHOLAS, L. E. \& BROOKSHIRE, R. H. (1993): “A system for quantifying the informativeness and efficiency of the connected speech in adults with aphasia", Journal of Speech and Hearing Research, 36, pp. 338-350.

PARADIS, M. (1977): "Bilingualism and aphasia", Whitaker, H. \& Whitaker, H. A. (eds.): Studies 
in Neurolinguistics, New York: Academic Press, pp. 65-121.

PARADIS, M. (1994): "Neurolinguistic aspects of implicit and explicit memory: Implications for bilingualism and second language acquisition", Ellis, N. C. (ed.): Implicit and explicit learning of languages, New York: Academic Press, pp. 393-419.

PARADIS, M. (2001): "Bilingual and polyglot aphasia", Boller, F. \& Grafman, J. (eds.): Handbook of Neuropsychology, Amsterdam: Elsevier, pp. 69-91.

PARADIS, M. (2004): A neurolinguistic theory of bilingualism, Amsterdam: John Benjamins.

PARADIS, M. \& LIBBEN, G. (1987): The assessment of bilingual aphasia, Hillsdale (NJ): Lawrence Erlbaum Associates.

PEÑA-CASANOVA, J. (1991): Test Barcelona. Normalidad, semiología y patología neuropsicológicas, Barcelona: Masson.

PERANI, D. \& ABUTALEBI, J. (2005): "The neural basis of first and second language processing", Current Opinion in Neurobiology, 15, pp. 202-206.

PERANI, D. et al. (1996): "Brain processing of native and foreign languages", Neuroreport, 7, pp. 2439-2444.

PISKE, T. et al. (2001): "Factors affectting degree of foreign accent in an L2: A review", Journal of Phonetics, 29, pp. 191-215.

PITRES, J. A. (1895): "Étude sur l'aphasie chez les polyglottes", Revue de médecine, 15, pp. 873-899. [Traducido en M. Paradis (ed.) (1983): Readings in aphasia in bilinguals and polyglots, Montreal: Didier, pp. 2649.]

RADMAN, N. et al. (2016): "The role of cognitive control system in recovery from bilingual apha-sia: A multiple single-case fMRI study", Neural Plasticity. Disponible en: https://www.ncbi.nlm.nih.gov /pmc/articles/PMC5124691/.

RIBOT, T. (1881): Les maladies de la mémoire, Paris: G. Baillière.

SCOVEL, T. (1988): A time to speak: A psycholinguistic inquiry into the critical period for human speech, New York: Newbury House Publishers.

SEBASTIAN-GALLÉS, N. et al. (2005): "The influence of initial exposure on lexical representation: Comparing early and simultaneous bilinguals", Journal of Memory and Language, 52, pp. 240-255.

SEBASTIÁN-GALLÉS, N. et al. (2006): "First- and second-language phonological representations in the mental lexicon", Journal of Cognitive Neuroscience, 18, pp. 1277-1291.

SIMMONDS, A. J. et al. (2011): “Two tongues, one brain: Imaging bilingual speech production", Frontiers in Psychology, 2, p. 166.

TAN, L. H. et al. (2003): "Neural systems of second language reading are shaped by native language", Human Brain Mapping, 18, pp. $158-166$.

ULLMAN, M. T. (2001a): "A neurocognitive perspective on language: The declarative/ procedural mo- 
del", Nature Reviews Neuroscience, 2, pp. 717-726.

ULLMAN, M. T. (2001b): "The neural basis of lexicon and grammar in first and second language: The declarative/procedural model", Bilingualism: Language and Cognition, 4, pp. 105-122.

WARTENBURGER, I. et al. (2003): "Early setting of grammatical processing in the bilingual brain", Neuron, 37, pp. 159170.

WEEKES, B. S. (ed.) (2010): Issues in bilingual aphasia, Hove: Psychology Press.
WEINREICH, U. (1953): Languages in contact, New York: Linguistic Circle of New York.

ZANINI, S. et al. (2010): "Spontaneous language production in bilingual Parkinsons disease patients: Evidence of greater phonological, morphological and syntactic impairments in native language", Brain and Language, 113, pp. 84-89.

ZANINI, S. et al. (2004): "Greater syntactic impairments in native language in bilingual Parkinsonian patients", Journal of Neurology, Neurosurgery and Psychiatry, 75, pp. 1678-1681. 